Supporting Information for Publication

\title{
Analytic Model of Nonequilibrium Charge Transport in Disordered Organic Semiconductors with Combined Energy and Off-Diagonal Disorder
}

\author{
Maxim D. Khan ${ }^{\dagger}$, Vladimir R. Nikitenko ${ }^{* *}$ and Oleg V. Prezhdo ${ }^{\ddagger *}$ \\ ${ }^{\dagger}$ National Research Nuclear University «MEPhI» (Moscow Engineering Physics Institute), \\ Kashirskoe shosse 31, 115409, Moscow, Russia \\ ${ }^{*}$ Department of Chemistry, University of Southern California, Los Angeles, CA 90089, United \\ States
}

*Corresponding authors. Email: vladronik@yandex.ru, prezhdo@usc.edu 


\section{DERIVATION OF THE EQUATION FOR THE CONCENTRATION OF CHARGE CARRIERS IN A NONEQUILIBRIUM TRANSPORT MODE, TAKING INTO ACCOUNT THE OFF-DIAGONAL DISORDER, AND ITS SOLUTION}

Let us write the balance equation of the multiple trapping model in the limit of low concentrations, i.e., at $\rho(E, \Gamma, x, t) \ll g(\Gamma, E)$, using eqs $2-4$, in the following form:

$$
\frac{\partial \rho(E, \Gamma, x, t)}{\partial t}=\tilde{\omega}(\Gamma)\langle\omega\rangle(E)\left[e^{-\frac{E}{k T}} g(E) \tilde{g}(\Gamma) \frac{p_{\mathrm{c}}(x, t)}{N_{\mathrm{c}}}-\rho(E, \Gamma, x, t)\right] .
$$

Let us introduce the demarcation energy, $E_{\mathrm{d}}(\Gamma, t)$, separating "shallow" and "deep" traps. We solve eq S1 for the case of "shallow" traps, $E>E_{\mathrm{d}}(\Gamma, t)$, in this case $\frac{\partial \rho(E, \Gamma, x, t)}{\partial t} \approx 0$, then in the zero approximation we get:

$$
\rho_{\mathrm{sh}}^{0}(E, \Gamma, x, t)=\frac{1}{N_{\mathrm{c}}} g_{\mathrm{sh}}(E) \tilde{g}(\Gamma) e^{-\frac{E}{k T}} p_{\mathrm{c}}(x, t),
$$

where

$$
g_{\text {sh }}(E)=\left\{\begin{array}{l}
g(E), E \geq E_{\mathrm{d}}(\Gamma, t), \\
0, E<E_{\mathrm{d}}(\Gamma, t),
\end{array}\right.
$$

and in the next (first) approximation of the perturbation theory, substituting eq S2 into S1, we obtain:

$$
\rho_{\mathrm{sh}}^{1}(E, \Gamma, x, t)=\frac{g_{\mathrm{sh}}(E) \tilde{g}(\Gamma)}{N_{\mathrm{c}}} e^{-\frac{E}{k T}} p_{\mathrm{c}}(x, t)-\frac{\tilde{g}(\Gamma)}{\tilde{\omega}(\Gamma)} \frac{g_{\mathrm{sh}}(E)}{N_{\mathrm{c}}} \frac{e^{-\frac{E}{k T}}}{\langle\omega\rangle(E)} \frac{\partial p_{\mathrm{c}}(x, t)}{\partial t} .
$$

In eq S1 for "deep" traps, $E<E_{d}(\Gamma, t)$, we neglect the 2 nd term on the right side (describes the release from traps), and we get:

$$
\begin{aligned}
& \frac{\partial \rho_{\mathrm{d}}(E, \Gamma, x, t)}{\partial t}=\frac{1}{N_{\mathrm{c}}} \tilde{\omega}(\Gamma) \tilde{g}(\Gamma)\langle\omega\rangle(E) g_{\mathrm{d}}(E) e^{-\frac{E}{k T}} p_{\mathrm{c}}(x, t), \\
& \rho_{\mathrm{d}}(E, \Gamma, x, t)=\frac{1}{N_{\mathrm{c}}} \tilde{\omega}(\Gamma)\langle\omega\rangle(E) \tilde{g}(\Gamma) g_{\mathrm{d}}(E) e^{-\frac{E}{k T}} \int_{0}^{t} p_{\mathrm{c}}\left(x, t^{\prime}\right) d t^{\prime},
\end{aligned}
$$

where

$$
g_{\mathrm{d}}(E)=\left\{\begin{array}{l}
0, E>E_{\mathrm{d}}(\Gamma, t) \\
g(E), E \leq E_{\mathrm{d}}(\Gamma, t)
\end{array}\right.
$$

Since

$$
\rho(E, \Gamma, x, t)=\rho_{\mathrm{sh}}(E, \Gamma, x, t)+\rho_{\mathrm{d}}(E, \Gamma, x, t),
$$


and

$$
p(x, t)=p_{\mathrm{c}}(x, t)+\int_{-\infty}^{\infty} \int_{-\infty}^{\infty} \rho(E, \Gamma, x, t) d E d \Gamma,
$$

then, taking into account eqs S4 and S6, we obtain:

$$
\begin{aligned}
& p(x, t)=p_{\mathrm{c}}(x, t)+\int_{-\infty}^{\infty} \tilde{g}(\Gamma) \int_{E_{\mathrm{d}}(\Gamma, t)}^{\infty} \frac{g(E)}{N_{\mathrm{c}}} e^{-\frac{E}{k T}} d E d \Gamma p_{\mathrm{c}}(x, t)- \\
& -\int_{-\infty}^{\infty} \frac{\tilde{g}(\Gamma)}{\tilde{\omega}(\Gamma)} \int_{E_{\mathrm{d}}(\Gamma, t)}^{\infty} \frac{g(E)}{N_{\mathrm{c}}} \frac{e^{-\frac{E}{k T}}}{\langle\omega\rangle(E)} d E d \Gamma \frac{\partial p_{\mathrm{c}}(x, t)}{\partial t}+ \\
& +\int_{-\infty}^{\infty} \tilde{g}(\Gamma) \tilde{\omega}(\Gamma) \int_{-\infty}^{E_{\mathrm{d}}(\Gamma, t)} \frac{g(E)}{N_{\mathrm{c}}}\langle\omega\rangle(E) e^{-\frac{E}{k T}} d E d \Gamma \int_{0}^{t} p_{\mathrm{c}}\left(x, t^{\prime}\right) d t^{\prime},
\end{aligned}
$$

or

$$
p(x, t)=\left[1+\frac{1}{\theta_{1}(t)}\right] p_{\mathrm{c}}(x, t)+\frac{1}{\tau(t)} \int_{0}^{t} p_{\mathrm{c}}\left(x, t^{\prime}\right) d t^{\prime}-\frac{1}{\theta_{2}(t)} \frac{\partial p_{\mathrm{c}}(x, t)}{\partial t},
$$

where

$$
\begin{aligned}
& \frac{1}{\theta_{1}(t)}=\int_{-\infty}^{\infty} \tilde{g}(\Gamma) \int_{E_{\mathrm{d}}(\Gamma, t)}^{\infty} \frac{g(E)}{N_{\mathrm{c}}} e^{-\frac{E}{k T}} d E d \Gamma, \\
& \frac{1}{\theta_{2}(t)}=\int_{-\infty}^{\infty} \frac{\tilde{g}(\Gamma)}{\tilde{\omega}(\Gamma)} \int_{E_{\mathrm{d}}(\Gamma, t)}^{\infty} \frac{g(E)}{N_{\mathrm{c}}} \frac{e^{-\frac{E}{k T}}}{\langle\omega\rangle(E)} d E d \Gamma, \\
& \frac{1}{\tau(t)}=\int_{-\infty}^{\infty} \tilde{g}(\Gamma) \tilde{\omega}(\Gamma) \int_{-\infty}^{E_{\mathrm{d}}(\Gamma, t)} \frac{g(E)}{N_{\mathrm{c}}}\langle\omega\rangle(E) e^{-\frac{E}{k T}} d E d \Gamma .
\end{aligned}
$$

Let us write the continuity equation, neglecting the conventional diffusion:

$$
\begin{aligned}
& \frac{\partial p(x, t)}{\partial t}+\frac{\partial j(x, t)}{\partial x}=0, \\
& j(x, t)=\mu_{\mathrm{c}} F p_{\mathrm{c}}(x, t) .
\end{aligned}
$$

Substitute eq S16 into eq S15:

$$
\frac{\partial p(x, t)}{\partial t}+\mu_{\mathrm{c}} F \frac{\partial p_{\mathrm{c}}(x, t)}{\partial x}=0 \text {. }
$$

We integrate eq S17 over time:

$$
p(x, t)-p(x, 0)+\mu_{\mathrm{c}} F \frac{\partial}{\partial x} \int_{0}^{t} p_{\mathrm{c}}\left(x, t^{\prime}\right) d t^{\prime}=0 .
$$

We transform eq S11, assuming that $\theta_{1}(t)<<1$, which is true for $\sigma / k T>>1$ : 


$$
\int_{0}^{t} p_{\mathrm{c}}\left(x, t^{\prime}\right) d t^{\prime}=\tau(t) p(x, t)-\frac{\tau(t)}{\theta_{1}(t)} p_{\mathrm{c}}(x, t)+\frac{\tau(t)}{\theta_{2}(t)} \frac{\partial p_{\mathrm{c}}(x, t)}{\partial t}
$$

and substitute it into eq S18, simultaneously multiplying by $\frac{\theta_{1}(t)}{\tau(t)}$ and transforming:

$$
\mu_{\mathrm{c}} \theta_{1}(t) F \frac{\partial}{\partial x} p(x, t)-\mu_{\mathrm{c}} F \frac{\partial p_{\mathrm{c}}(x, t)}{\partial x}+\mu_{\mathrm{c}} F \frac{\theta_{1}(t)}{\theta_{2}(t)} \frac{\partial^{2} p_{\mathrm{c}}(x, t)}{\partial t \partial x}=-\frac{\theta_{1}(t)}{\tau(t)}(p(x, t)-p(x, 0)) .
$$

Introducing the following notation:

$$
\begin{aligned}
& \mu(t)=\mu_{c} \theta_{1}(t), \\
& t_{\text {rel }}(t)=\frac{\theta_{1}(t)}{\theta_{2}(t)}, \\
& \lambda(t)=\frac{\theta_{1}(t)}{\tau(t)},
\end{aligned}
$$

we get:

$$
\mu(t) F \frac{\partial p(x, t)}{\partial x}-\mu_{\mathrm{c}} F \frac{\partial p_{\mathrm{c}}(x, t)}{\partial x}+t_{\text {rel }}(t) \frac{\partial}{\partial t}\left(\mu_{\mathrm{c}} F \frac{\partial p_{\mathrm{c}}(x, t)}{\partial x}\right)=-\lambda(t)(p(x, t)-p(x, 0)) .
$$

Eliminating $p_{c}(x, t)$ from eq S24 and using eq S17 one obtains:

$$
\frac{\partial p(x, t)}{\partial t}+\mu(t) F \frac{\partial p(x, t)}{\partial x}-t_{\text {rel }}(t) \frac{\partial^{2} p(x, t)}{\partial t^{2}}=-\lambda(t)(p(x, t)-p(x, 0)) .
$$

Let us approximate $\frac{\partial^{2} p(x, t)}{\partial t^{2}}$ from the perturbation theory. In the zero order approximation, assuming that $\frac{\partial^{2} p(x, t)}{\partial t^{2}} \approx 0$, from eq S25 we obtain:

$$
\frac{\partial p(x, t)}{\partial t}=-\mu(t) F \frac{\partial p(x, t)}{\partial x}-\lambda(t)(p(x, t)-p(x, 0))
$$

Time differentiation of eq S26 gives:

$$
\begin{aligned}
& \frac{\partial^{2} p(x, t)}{\partial t^{2}} \approx-\mu(t) F \frac{\partial}{\partial x} \frac{\partial p(x, t)}{\partial t}-F \frac{\partial \mu(t)}{\partial t} \frac{\partial p(x, t)}{\partial x}- \\
& -\lambda(t) \frac{\partial p(x, t)}{\partial t}-\frac{\partial \lambda(t)}{\partial t}(p(x, t)-p(x, 0)) .
\end{aligned}
$$

Substitute eq S26 into eq S27:

$$
\begin{aligned}
& \frac{\partial^{2} p(x, t)}{\partial t^{2}} \approx \mu^{2}(t) F^{2} \frac{\partial^{2} p(x, t)}{\partial x^{2}}+\mu(t) F \lambda(t)\left(\frac{\partial p(x, t)}{\partial x}-\frac{\partial p(x, 0)}{\partial x}\right)-F \frac{\partial \mu(t)}{\partial t} \frac{\partial p(x, t)}{\partial x}+ \\
& +\lambda(t) \mu(t) F \frac{\partial p(x, t)}{\partial x}+\lambda^{2}(t)(p(x, t)-p(x, 0))-\frac{\partial \lambda(t)}{\partial t}(p(x, t)-p(x, 0)) .
\end{aligned}
$$


Neglecting in right-hand side of eq S28 all terms except the first, which is true for sufficiently long times, we get instead of eq S25:

$$
\frac{\partial p(x, t)}{\partial t}+\mu(t) F \frac{\partial p(x, t)}{\partial x}-\mu^{2}(t) F^{2} t_{\text {rel }}(t) \frac{\partial^{2} p(x, t)}{\partial x^{2}}=-\lambda(t)(p(x, t)-p(x, 0)),
$$

and introducing the following notation:

$$
D_{\mathrm{F}}(t)=\mu^{2}(t) F^{2} t_{\text {rel }}(t) \text {, }
$$

$D_{\mathrm{F}}(t)$ is the field-assisted diffusion coefficient, we obtain the drift-diffusion equation for nonequilibrium transport (it is the eq 12 in the main text):

$$
\frac{\partial p(x, t)}{\partial t}+\mu(t) F \frac{\partial p(x, t)}{\partial x}-D_{F}(t) \frac{\partial^{2} p(x, t)}{\partial x^{2}}=-\lambda(t)(p(x, t)-p(x, 0))
$$

Let us solve eq S31 with the initial condition

$$
p(x, 0)=\sigma_{0} \delta(x),
$$

$\sigma_{0}$ is an initial area density of photogenerated carriers.

For typical conditions of a time-of-flight experiment, the field can be considered strong enough to neglect ordinary diffusion and, consequently, the influence of the electrode at $x=0$ on the motion of carriers. On the other hand, the opposite electrode (at $x=L$ ) does not interfere with the drain of carriers, i.e., it also does not disturb their motion. Therefore, we are looking for a solution to eq S31 in an infinite environment. Applying the Fourier transform,

$$
\begin{aligned}
& p(k, t)=\int_{-\infty}^{\infty} e^{-i k x} p(x, t) d x, \text { we obtain }{ }^{1} \\
& p(x, t)=G(x, t, 0)+\int_{0}^{t} \lambda\left(t^{\prime}\right) G\left(x, t, t^{\prime}\right) d t^{\prime},
\end{aligned}
$$

where

$$
\begin{aligned}
& G\left(x, t, t^{\prime}\right)=\frac{\sigma_{0}}{\sqrt{4 \pi \tilde{D}_{\mathrm{F}}\left(t, t^{\prime}\right)}} e^{-\Lambda\left(t, t^{\prime}\right)-\frac{\left(x-M\left(t, t^{\prime}\right)\right)^{2}}{4 \tilde{D}_{\mathrm{F}}\left(t, t^{\prime}\right)}} \\
& M\left(t, t^{\prime}\right)=F \int_{t^{\prime}}^{t} \mu\left(t^{\prime}\right) d t^{\prime} \\
& \tilde{D}_{\mathrm{F}}\left(t, t^{\prime}\right)=\int_{t^{\prime}}^{t} D_{\mathrm{F}}\left(t^{\prime}\right) d t^{\prime} \\
& \Lambda\left(t, t^{\prime}\right)=\int_{t^{\prime}}^{t} \lambda\left(t^{\prime}\right) d t^{\prime}
\end{aligned}
$$




\section{CALCULATION OF THE TRANSIENT CURRENT FOR THE CONDITIONS OF THE TIME-OF-FLIGHT EXPERIMENT}

Find $j(t)$ from eq S16, integrating it over $x$ and multiplying by $\frac{e}{L}$

$$
j(t)=\frac{e}{L} \int_{0}^{L} j(x, t) d x=\frac{e \mu_{\mathrm{c}} F}{L} \int_{0}^{L} p_{\mathrm{c}}(x, t) d x .
$$

We multiply eq S17 by $\frac{e}{L} x$ and integrate over $x$

$$
\frac{e}{L} \frac{\partial}{\partial t} \int_{0}^{L} x p(x, t) d x+\frac{e}{L} \mu_{\mathrm{c}} F \int_{0}^{L} x \frac{\partial p_{\mathrm{c}}(x, t)}{\partial x} d x=0,
$$

considering that

$$
\begin{aligned}
& \int_{0}^{L} x \frac{\partial p_{\mathrm{c}}(x, t)}{\partial x} d x=\left.x p_{\mathrm{c}}(x, t)\right|_{0} ^{L}-\int_{0}^{L} p_{\mathrm{c}}(x, t) d x=L p_{\mathrm{c}}(L, t)-\int_{0}^{L} p_{\mathrm{c}}(x, t) d x, \\
& \text { get } \\
& \frac{e \mu_{\mathrm{c}} F}{L} \int_{0}^{L} p_{\mathrm{c}}(x, t) d x=\frac{e}{L} \frac{\partial}{\partial t} \int_{0}^{L} x p(x, t) d x+e \mu_{\mathrm{c}} F p_{\mathrm{c}}(L, t) .
\end{aligned}
$$

Substitute eq S41 into eq S38

$$
j(t)=\frac{e}{L} \frac{\partial}{\partial t} \int_{0}^{L} x p(x, t) d x+e \mu_{\mathrm{c}} F p_{\mathrm{c}}(L, t) .
$$

We multiply eq S17 by $L$ and integrate over $x$

$$
e \frac{\partial}{\partial t} \int_{0}^{L} p(x, t) d x+e \mu_{\mathrm{c}} F \int_{0}^{L} \frac{\partial p_{\mathrm{c}}(x, t)}{\partial x} d x=0,
$$

considering that

$$
\int_{0}^{L} \frac{\partial p_{\mathrm{c}}(x, t)}{\partial x} d x=\left.p_{\mathrm{c}}(x, t)\right|_{0} ^{L}=p_{\mathrm{c}}(L, t)-p_{\mathrm{c}}(0, t),
$$

get

$$
e \mu_{\mathrm{c}} F p_{\mathrm{c}}(L, t)=-e \frac{\partial}{\partial t} \int_{0}^{L} p(x, t) d x+e \mu_{\mathrm{c}} F p_{\mathrm{c}}(0, t) .
$$

Substitute eq S45 into eq S42

$$
j(t)=\frac{e}{L} \frac{\partial}{\partial t} \int_{0}^{L}(x-L) p(x, t) d x+e \mu_{c} F p_{\mathrm{c}}(0, t) .
$$

Using the boundary condition

$$
j(0, t)=0,
$$

from eq $\mathrm{S} 17$ we get

$$
p_{\mathrm{c}}(0, t)=0 \text {, }
$$


then

$$
j(t)=\frac{e}{L} \frac{\partial}{\partial t} \int_{0}^{L}(x-L) p(x, t) d x .
$$

Substitute eq S33 into eq S49, integrate and obtain

$$
j(t)=\frac{e \sigma_{0}}{2 L}\left(\int_{0}^{t} \lambda\left(t^{\prime}\right) J\left(t, t^{\prime}\right) d t^{\prime}+J(t, 0)-2 L \lambda(t)\right)
$$

where

$$
J\left(t, t^{\prime}\right)=e^{-\Lambda\left(t, t^{\prime}\right)}\left(\begin{array}{l}
\left(\mu(t) F+\left(L-M\left(t, t^{\prime}\right)\right) \lambda(t)\right) \operatorname{erfc}\left(-\frac{L-M\left(t, t^{\prime}\right)}{2 \sqrt{\tilde{D}_{\mathrm{F}}\left(t, t^{\prime}\right)}}\right)+ \\
+\frac{2 \tilde{D}_{\mathrm{F}}\left(t, t^{\prime}\right) \lambda(t)-D_{\mathrm{F}}(t)}{\sqrt{\pi \tilde{D}_{\mathrm{F}}\left(t, t^{\prime}\right)}} e^{-\frac{\left(L-M\left(t, t^{\prime}\right)\right)^{2}}{4 \tilde{D}_{\mathrm{F}}\left(t, t^{\prime}\right)}}
\end{array}\right) .
$$

In eq S51 terms are discarded which are small under the condition $\sqrt{\tilde{D}_{\mathrm{F}}(t, 0)}<<M(t, 0)$, i.e. the dispersion from the FAD diffusion is much smaller than the drift shift, which is a condition of applicability of the FAD diffusion (obtained from perturbation theory). ${ }^{1}$

\section{DETERMINATION OF THE EFFECTIVE CONCENTRATION OF "CONDUCTING" STATES, $N_{\mathrm{c}}$.}

Let us determine the value $N_{\mathrm{c}}$ in eq $\mathrm{S} 1$ or 3 in the main text. One has to note that in the MTR model

$$
\mu=\mu_{\mathrm{c}} \frac{p_{\mathrm{c}}(x, t)}{p(x, t)},
$$

where

$$
\mu_{\mathrm{c}}=\frac{e}{k T} a^{2} \frac{1}{t_{0}} \text {. }
$$

The "conducting" states assumed to be in quasi-equilibrium hence their occupation probability does not depend on the parameter $\Gamma$, from the thermodynamic reasoning. Using eq A3 and the definition of a function $\varphi(E, \Gamma)$ as a probability that a state with parameters $E, \Gamma$ is "conductive" we can write

$$
p_{\mathrm{c}}(x, t)=\frac{p_{\mathrm{c}}(x, t)}{N_{\mathrm{c}}} \int_{-\infty}^{\infty} \int_{-\infty}^{\infty} g(\Gamma, E) \varphi(\Gamma, E) e^{-\frac{E}{k T}} d \Gamma d E,
$$

where

$$
\varphi(E, \Gamma)=\left(\frac{k T}{W}\right)^{\eta}\left(1-e^{-\omega(E, \Gamma) t_{0}}\right) \approx\left(\frac{k T}{W}\right)^{\eta} \omega(E, \Gamma) t_{0},
$$


One can take the time $t_{0}$ as very small value, if the respective state belongs to the percolation claster. In eq S55 $\left(\frac{k T}{W}\right)^{\eta}$ is the relative density of the percolation cluster, $W$ is the width of the distribution function, $\chi$ is the number (critical index of the correlation radius of the percolation cluster), ${ }^{2,3}$ close to unity.

Eliminating $p_{\mathrm{c}}(x, t)$ from eq S55 and using eqs 2, 4, 7 and S55, one obtains

$$
\frac{N_{\mathrm{c}}}{N} \approx t_{0} \int_{-\infty}^{\infty} d E \frac{g(E)}{N}\langle\omega\rangle(E) \exp \left(-\frac{E}{k T}\right) \approx v_{0} t_{0} \frac{k T}{\sqrt{2} \sigma} \exp \left(-\frac{E_{\mathrm{tr}}}{k T}\right)
$$

i.e., the off-diagonal disorder does not have an effect on the value of $N_{\mathrm{c}}$.

\section{CALCULATION OF THE MOBILITY AND FIELD DIFFUSION COEFFICIENT FOR THE GAUSSIAN DISTRIBUTION OF TRAPS}

Considering eqs S12-S14, S53, S56 for S21-S23 we get

$$
\begin{aligned}
& \mu(t)=\frac{e}{k T} a^{2}\left(\frac{k T}{W}\right)^{\eta} \frac{\int_{-\infty}^{\infty} \tilde{g}(\Gamma) \tilde{\omega}(\Gamma) d \Gamma \int_{-\infty}^{\infty} g(E)\langle\omega\rangle(E) e^{-\frac{E}{k T}} d E}{\int_{-\infty}^{\infty} \tilde{g}(\Gamma) \int_{E_{d}(\Gamma, t)}^{\infty} g(E) e^{-\frac{E}{k T}} d E d \Gamma}, \\
& t_{\text {rel }}(t)=\frac{\int_{-\infty}^{\infty} \frac{\tilde{g}(\Gamma)}{\tilde{\omega}(\Gamma)} \int_{E_{d}(\Gamma, t)}^{\infty} \frac{g(E) e^{-\frac{E}{k T}}}{\langle\omega\rangle(E)} d E d \Gamma}{\int_{-\infty}^{\infty} \tilde{g}(\Gamma) \int_{E_{d}(\Gamma, t)}^{\infty} g(E) e^{-\frac{E}{k T}} d E d \Gamma}, \\
& \lambda(t)=\frac{\int_{-\infty}^{\infty} \tilde{g}(\Gamma) \tilde{\omega}(\Gamma) \int_{-\infty}^{E_{d}(\Gamma, t)} g(E)\langle\omega\rangle(E) e^{-\frac{E}{k T}} d E d \Gamma}{\tilde{g}(\Gamma) \int_{E_{d}(\Gamma, t)}^{\infty} g(E) e^{-\frac{E}{k T}} d E d \Gamma} .
\end{aligned}
$$

Remarkable that the results of eqs S57-S59 does not depend on the value of the critical time $t_{0}$. Note that $E_{\mathrm{d}}(\Gamma, t) \rightarrow-\infty$ at $t \rightarrow \infty$, and we obtain the following quasi-equilibrium values:

$$
\mu_{\mathrm{eq}}=\frac{e}{k T} a^{2}\left(\frac{k T}{W}\right)^{\eta} \frac{\int_{-\infty}^{\infty} \tilde{g}(\Gamma) \tilde{\omega}(\Gamma) d \Gamma \int_{-\infty}^{\infty} g(E)\langle\omega\rangle(E) e^{-\frac{E}{k T}} d E}{\int_{-\infty}^{\infty} \tilde{g}(\Gamma) d \Gamma},
$$


$t_{\text {rel eq }}=\frac{\int_{-\infty}^{\infty} \frac{\tilde{g}(\Gamma)}{\tilde{\omega}(\Gamma)} d \Gamma \int_{-\infty}^{\infty} \frac{g(E) e^{-\frac{E}{k T}}}{\langle\omega\rangle(E)} d E}{\int_{-\infty}^{\infty} \tilde{g}(\Gamma) d \Gamma \int_{-\infty}^{\infty} g(E) e^{-\frac{E}{k T}} d E}$.

We further assume that

$$
\begin{aligned}
& g(E)=\frac{N}{\sqrt{2 \pi} \sigma} e^{-\frac{E^{2}}{2 \sigma^{2}},} \\
& W=\sqrt{2} \sigma, \\
& \eta \approx 1, \\
& \tilde{g}(\Gamma)=\frac{1}{\sqrt{2 \pi} \Sigma} e^{-\frac{\left(\Gamma-\Gamma_{\mathrm{m}}\right)^{2}}{2 \Sigma^{2}}}, \\
& \Gamma_{\mathrm{m}}=\frac{\Sigma^{2}}{2}+\langle\Gamma\rangle, \\
& \tilde{\omega}(\Gamma)=e^{-(\Gamma-\langle\Gamma\rangle)}, \\
& \langle\Gamma\rangle=\frac{E_{\mathrm{tr}}}{k T}, \\
& \langle\omega\rangle(E)=\left\{\begin{array}{l}
v_{0} e^{-\frac{E_{\mathrm{tr}}}{k T}}, E>E_{\mathrm{tr}}, \\
v_{0} e^{-\frac{E_{\mathrm{tr}}-E}{k T}}, E \leq E_{\mathrm{tr}} .
\end{array}\right.
\end{aligned}
$$

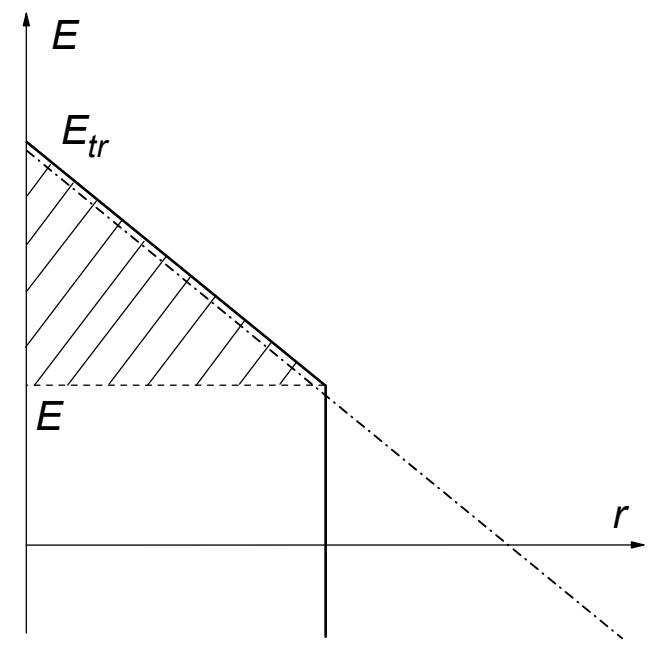

Figure S1. The area of integration for calculating the effective transport level $E_{\mathrm{tr}}$. The "physical" transport level, see the solid line. The dash-dotted line corresponds to the case of $E \rightarrow-\infty$. 
Let us define the effective transport level $E_{\text {tr }}$, as in refs. 4 and 5 , from the condition $n_{0}\left(E_{\mathrm{tr}}\right)=B$, in other terms, $n_{0}(\langle\Gamma\rangle)=B, B \approx 2.77$. The mean number of target neighbor sites for the initial state of the energy $E$, whose hopping rates are not smaller than a given value of $\omega=v_{0} \exp (-u), n_{0}(u, E)$, is calculated by integrating the DOS $g(E)$ over the area of $r-E$ space, which is shown in Figure S1. One obtains

$$
\frac{4 \pi}{3}\left(\frac{u}{2 \gamma}\right)^{3} \int_{-\infty}^{E} g\left(E^{\prime}\right) d E^{\prime}+\frac{\pi}{6 \gamma^{3}} \int_{E}^{E+u k T} g\left(E^{\prime}\right)\left[\frac{E+u k T-E^{\prime}}{k T}\right]^{3} d E^{\prime}=B .
$$

The first term in eq S70, which corresponds to the downward in energy jumps, is negligible if $E \rightarrow-\infty$. We obtain the equation for the effective transport level $E_{\mathrm{tr}}$ from eq S70, denoting $E_{t r}=E+u k T$ and providing that $E \rightarrow-\infty:^{4}$

$$
\frac{4 \pi}{3} N \int_{-\infty}^{E_{\mathrm{tr}}} g(E)\left(\frac{E_{\mathrm{tr}}-E}{2 \gamma k T}\right)^{3} d E=B
$$

One has to calculate the probability that a carrier does not return to the initial state of the energy $E$ after first jump on the state of the energy $E^{\prime}$, see eq A6. Following the way of the ref. 6 and introducing the parameter $\Gamma$ as $\Gamma=u+E / k T$, we can see that

$$
n(E, u)=n(\Gamma)=\frac{4 \pi}{(2 \gamma)^{3}} \int_{-\infty}^{\Gamma k T} d E^{\prime} g\left(E^{\prime}\right) \int_{0}^{\Gamma-E^{\prime} k T} d y y^{2}\left[1-\exp \left(-n_{b}\left(E^{\prime}, y\right)\right)\right],
$$

where $y=2 \gamma r$,

$$
n_{b}\left(\varepsilon^{\prime}, y\right)=\frac{\pi N y^{3}}{96 \gamma^{3}}\left\{\frac{11}{12} \operatorname{erfc}\left(-\varepsilon^{\prime}\right)+\int_{\varepsilon^{\prime}}^{\varepsilon^{\prime}+\alpha y} d z \frac{e^{-z^{2}}}{\sqrt{\pi}}\left[8 \frac{\left(\varepsilon^{\prime}+\alpha y-z\right)}{\alpha y}+3\left(\frac{\varepsilon^{\prime}+\alpha y-z}{\alpha y}\right)^{4}\right]\right\},
$$

where $\varepsilon^{\prime}=E^{\prime} / \sqrt{2} \sigma, \alpha=k T / \sqrt{2} \sigma$.

One obtains from eqs $\mathrm{S} 72$ and A6

$$
P\left(u, E, E^{\prime}\right)=P\left(\Gamma, E^{\prime}\right)=3\left(\Gamma-E^{\prime} / k T\right)^{-3} \int_{0}^{\Gamma-E^{\prime} / k T} d y y^{2}\left[1-\exp \left(-n_{b}\left(E^{\prime}, y\right)\right)\right] .
$$

Let us define demarcation energy $E_{d}(\Gamma, t)$ :

$$
\tilde{\omega}(\Gamma)\langle\omega\rangle\left(E_{\mathrm{d}}(\Gamma, t)\right) t=1
$$

i.e.

$$
e^{-\Gamma} v_{0} e^{\frac{E_{\mathrm{d}}(\Gamma, t)}{k T}} t=1,
$$

hence

$$
E_{\mathrm{d}}(\Gamma, t)=k T\left(\Gamma-\ln \left(v_{0} t\right)\right) .
$$


Taking into account eqs S62 - S69, and also performing integration where possible, one obtains:

$$
\begin{aligned}
& \mu(t)=\frac{e}{k T} a^{2} v_{0} e^{-\frac{E_{\mathrm{tr}}}{k T}} \frac{k T}{\sqrt{2} \sigma} \frac{\left(e^{-\left(\frac{\sigma}{\sqrt{2} k T}\right)^{2}} \operatorname{erfc}\left(-\frac{E_{\mathrm{tr}}}{\sqrt{2} \sigma}\right)+\operatorname{erfc}\left(\frac{E_{\mathrm{tr}}}{\sqrt{2} \sigma}+\frac{\sigma}{\sqrt{2} k T}\right)\right)}{\int_{-\infty}^{\infty} \frac{e^{-\frac{\left(\Gamma-\Gamma_{\mathrm{m}}\right)^{2}}{2 \Sigma^{2}}}}{\sqrt{2 \pi} \Sigma} \operatorname{erfc}\left(\frac{E_{\mathrm{d}}(\Gamma, t)}{\sqrt{2} \sigma}+\frac{\sigma}{\sqrt{2} k T}\right) d \Gamma}, \\
& t_{\text {rel }}(t)=\frac{e^{3\left(\frac{\sigma}{\sqrt{2} k T}\right)^{2}} \int_{-\infty}^{\Gamma_{\max }(t)} e^{-\frac{\left(\Gamma-\Gamma_{\mathrm{m}}\right)^{2}}{2 \Sigma^{2}}+\Gamma}\left(\operatorname{erfc}\left(-\frac{E_{\mathrm{tr}}}{\sqrt{2} \sigma}-\frac{\sqrt{2} \sigma}{k T}\right)-\operatorname{erfc}\left(-\frac{E_{\mathrm{d}}(\Gamma, t)}{\sqrt{2} \sigma}-\frac{\sqrt{2} \sigma}{k T}\right)\right) d \Gamma}{v_{0} e^{-\frac{E_{\mathrm{tr}}}{k T}} \int_{-\infty}^{\infty} e^{-\frac{\left(\Gamma-\Gamma_{\mathrm{m}}\right)^{2}}{2 \Sigma^{2}}} \operatorname{erfc}\left(\frac{E_{\mathrm{d}}(\Gamma, t)}{\sqrt{2} \sigma}+\frac{\sigma}{\sqrt{2} k T}\right) d \Gamma}+ \\
& +\frac{e^{\Sigma^{2}} \operatorname{erfc}\left(\frac{E_{\mathrm{tr}}}{\sqrt{2} \sigma}+\frac{\sigma}{\sqrt{2} k T}\right)}{v_{0} e^{-\frac{E_{\mathrm{tr}}}{k T}} \int_{-\infty}^{\infty} \frac{e^{-\frac{\left(\Gamma-\Gamma_{\mathrm{m}}\right)^{2}}{2 \Sigma^{2}}}}{\sqrt{2 \pi \Sigma}} \operatorname{erfc}\left(\frac{E_{\mathrm{d}}(\Gamma, t)}{\sqrt{2} \sigma}+\frac{\sigma}{\sqrt{2} k T}\right) d \Gamma}, \\
& \lambda(t)=\frac{v_{0} e^{-\left(\frac{\sigma}{\sqrt{2} k T}\right)^{2} \Gamma_{\max }(t)} \int_{-\infty}^{-\frac{\left(\Gamma-\Gamma_{\mathrm{m}}\right)^{2}}{2 \Sigma^{2}}-\Gamma} \operatorname{erfc}\left(-\frac{E_{\mathrm{d}}(\Gamma, t)}{\sqrt{2} \sigma}\right) d \Gamma}{\int_{-\infty}^{\infty} e^{-\frac{\left(\Gamma-\Gamma_{\mathrm{m}}\right)^{2}}{2 \Sigma^{2}}} \operatorname{erfc}\left(\frac{E_{\mathrm{d}}(\Gamma, t)}{\sqrt{2} \sigma}+\frac{\sigma}{\sqrt{2} k T}\right) d \Gamma}, \\
& \mu_{e q}=\frac{e}{\sqrt{2} \sigma} a^{2} v_{0} e^{-\frac{E_{\mathrm{tr}}}{k T}} \frac{1}{2}\left(e^{-\left(\frac{\sigma}{\sqrt{2} k T}\right)^{2}} \operatorname{erfc}\left(-\frac{E_{\mathrm{tr}}}{\sqrt{2} \sigma}\right)+\operatorname{erfc}\left(\frac{E_{\mathrm{tr}}}{\sqrt{2} \sigma}+\frac{\sigma}{\sqrt{2} k T}\right)\right) \text {, } \\
& t_{\text {rel eq }}=\frac{e^{\Sigma^{2}+\frac{E_{\mathrm{tr}}}{k T}}}{2 v_{0}}\left(e^{\frac{3}{2}\left(\frac{\sigma}{k T}\right)^{2}} \operatorname{erfc}\left(-\frac{E_{\mathrm{tr}}}{\sqrt{2} \sigma}-\frac{\sqrt{2} \sigma}{k T}\right)+\operatorname{erfc}\left(\frac{E_{\mathrm{tr}}}{\sqrt{2} \sigma}+\frac{\sigma}{\sqrt{2} k T}\right)\right) .
\end{aligned}
$$

We define $\Gamma_{\max }$ from the condition $E_{\mathrm{d} \max }(\Gamma, t)=E_{\mathrm{tr}}$ :

$\Gamma_{\max }(t)=\frac{E_{\mathrm{tr}}}{k T}+\ln \left(v_{0} t\right)$. 


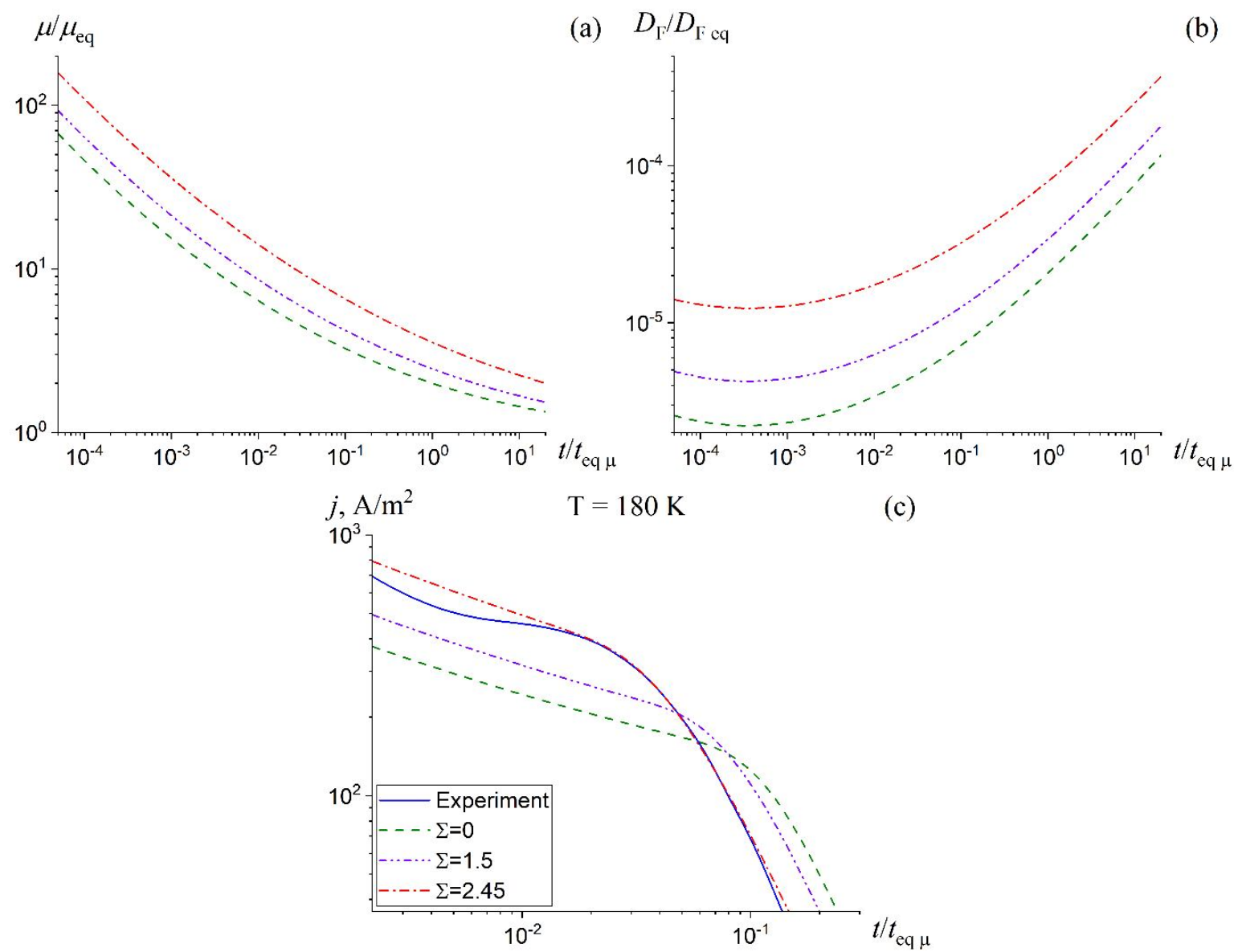

Figure S2. Dependence of mobility (a), field diffusion coefficient (b), and transient current (c) on the off-diagonal disorder parameter, $\Sigma$, for temperature, $T, 180 \mathrm{~K}$. 


\section{REFERENCES}

(1) Nikitenko, V. R.; Seggern, H. von; Bässler, H. Non-Equilibrium Transport of Charge Carriers in Disordered Organic Materials. J. Phys. Condens. Matter 2007, 19, 136210.

(2) Shklovskii, B. I.; Efros, A. L. Electronic Properties of Doped Polyacetylene, 1st ed.; Springer: Berlin, 1984.

(3) Cottaar, J.; Coehoorn, R.; Bobbert, P. A. Scaling Theory for Percolative Charge Transport in Molecular Semiconductors: Correlated versus Uncorrelated Energetic Disorder. Phys. Rev. B 2012, 85, 245205.

(4) Nikitenko, V. R.; Strikhanov, M. N. Transport Level in Disordered Organics: An Analytic Model and Monte-Carlo Simulations. J. Appl. Phys. 2014, 115, 073704.

(5) Khan, M. D.; Nikitenko, V. R.; Tyutnev, A. P.; Ikhsanov, R. S. Joint Application of Transport Level and Effective Temperature Concepts for an Analytic Description of the Quasi- and Nonequilibrium Charge Transport in Disordered Organics. J. Phys. Chem. C 2019, 123, 1653-1659.

(6) Arkhipov, V. I.; Emelianova, E. V.; Adriaenssens, G. J. Effective Transport Energy versus the Energy of Most Probable Jumps in Disordered Hopping Systems. Phys. Rev. B 2001, $64,125125$. 